\title{
Nonstandard analysis and the theory of shape*
}

by

Frank Wattenberg (Amherst, Mass.)

Abstract. This paper develops and exploits an approach to the theory of shape using the 列 bedding of $X$ into the normed linear space $Y$ such that $e(X)$ is closed in its convex hull. Let * $Y$ be an bedding of $X$ into the normed linear space $Y$ such that $e(X)$ is closed in its convex hull. $y$ at $y \in{ }^{*} Y$ such that $y$ is infinitely app to $* X$ Then one can apply essentially the usual homology and homotopy theory in * $Y$ to closed to *X. Then one paper $\mathcal{E}(X)$. This approach produces results very much like those obtained using shape then applies these techniques to obtain a classification theorem for covering spaces without the usual local connectivity assumptions on the base space. The result obtained is analogous to that obtained by Fox in his paper, On shape [6].

Consider the "Warsaw Circle",

$$
\begin{aligned}
K=(\{0\} \times[-2,1]) \cup([0,1] \times\{-2\}) \cup(\{1\} \times[-2, \sin 1]) \cup \\
\cup\{(x, \sin (1 / x)) \mid 0<x \leqslant 1\},
\end{aligned}
$$

picture below.

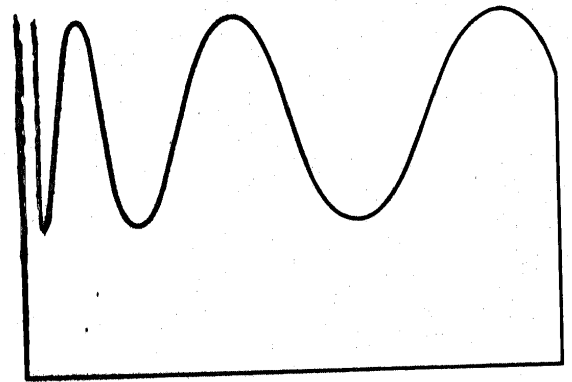

The usual functors of algebraic topology are not very useful for this space. For example, all its singular homology groups are trivial despite the fact that it disconnects $\boldsymbol{R}^{2}$ so that Alexander duality [14, Theorem 6.2.16] would seem to indicate

* This paper was presented at the summer 1974 Oberwolfach Conference on Nonstandard Analysis, dedicated to the memory of Abraham Robinson. 
that $H_{1}(K)=Z$. Similarly, the fundamental group of $K$ is trivial although its covering spaces are entirely analogous to those of the circle, which indicated that $\pi_{1}(K)$ should also be $Z$. In fact, $K$ behaves in many ways as if it had the same homotopy type as the circle, $S$.

This situation has been resolved for some time in homology theory by the use of Čech or Vietoris homology theory. D. E. Christie in [3] developed analogous techniques for studying the homotopy groups of such spaces. However, it is only in the past few years that Borsuk [1], [2]; Fox [6] and others have developed and exploited systematic homotopy theoretic techniques for investigating spaces like this. In particular, Borsuk's notion of shape is a natural variant of homotopy type by which $S$ and $K$ have the same shape. In [11] McCord used nonstandard analysis to provide a natural approach to a Vietoris-like homology theory.

The main idea of the present paper is to obtain a unificd approach to the "algebraic topology" of spaces like $K$ by interposing a natural nonstandard topological functor between topological spaces and the usual functors of algebraic topology. For example, let $\mathscr{E}(K)$ denote the set of all points in a nonstandard model $* R^{2}$ of $\boldsymbol{R}^{2}$ which are infinitely close to $K$.

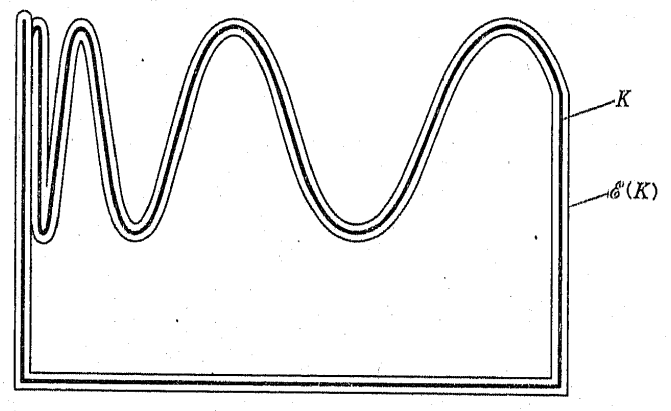

If we define $\pi_{1}(\mathscr{E}(K))$ to be the group formed by the (external) set of equivalence classes of internal *continuous maps $* S \rightarrow \mathscr{E}(K)$ with the usual homotopy relation and group operation, then it is straightforward to check that $\pi_{1}(\mathscr{E}(K)) \cong{ }^{*} Z$ exactly as one would expect from studying the covering spaces of $K$.

Throughout this paper we will deal only with metric spaces. When several spaces $X_{1}, X_{2}, \ldots$ are being considered, their nonstandard extensions will all be taken in a single nonstandard model $* \mathscr{M}$. That is, we let $\mathscr{M}$ denote the complete higher order structure on $X=\bigcup X_{i}$ and let * $\mathscr{M}$ be a higher order elementary extension of $\mathscr{M},[10],[13]$. The sets $* X_{1}, * X_{2}, \ldots$ will then all live in $* \mathscr{M}$. If $P$ denotes an object in $\mathscr{M}$ then the corresponding object in $* \mathscr{M}$ will be denoted by $* P$. In particular, an internal function $f:{ }^{*} X \rightarrow{ }^{*} Y$ will be said to be *continuous if it is in ${ }^{*} C(X, Y)$ where $C(X, Y)$ is the (standard) set of all continuous functions $g: X \rightarrow Y$. We will always take $* \mathscr{M}$ to be $x^{+}$saturated where $x$ is any cardinal larger than the cardinality of the universe of $\mathscr{M}$, although many of our arguments go through for weaker nonstandard models. If $X$ is a topological space and $x \in X$, the monad of $x$ in $* X$, denoted $\mu(x)$, is given by $\mu(x)=\{y \mid * d(x, y)$ is infinitesimal $\}$. If $y \in * X$, and for some $x \in X, y \in \mu(x)$ we say $y$ is nearstandard and write $x=\operatorname{St}(y)$. Notice, $\operatorname{St}(y)$ is necessarily unique.

In the first section of this paper we define a category $\mathscr{E}$ which is convenient to work in. The "envelope" functor we wish to interpose between topological spaces and the usual functors of algebraic topology is developed in the second section. Finally, in the last section we use this technique to obtain a classification of covering spaces $p: E \rightarrow B$ in terms of $\pi_{1}(\mathscr{E}(B))$ without the usual connectivity conditions on $B$. This result is analogous to Fox's classification [6] in terms of the fundamental trope of $B$.

I. The category $\mathscr{E}$. Throughout this paper we will be working with external sets and mappings which we want to behave as much as possible like internal sets and mappings. The category $\mathscr{E}$ defined in this section seems to be appropriate for our purposes.

I. 1. Definition. An $\mathscr{E}$-set is a subset $A$ (frequently external) of a nonstandard model $* X$ of a topological space $X$. If $A \subseteq * X$ and $B \subseteq * Y$ are two $\mathscr{E}$-sets a mapping $f: A \rightarrow B$ is said to be an $\mathscr{E}$-map provided for every internal set $C \subseteq A,\left.f\right|_{C}$ is internal and *continuous. Let $\mathscr{E}$ denote the category of $\mathscr{E}$-sets and $\mathscr{E}$-maps. (It is straightforward to verify that $\mathscr{E}$ is a category.)

Notice, in particular, that if $X$ is a topological space and $A$ is an internal subset of $* X$ then $A$ is an $\mathscr{E}$-set. If $B \subseteq^{*} Y$ is another $\mathscr{E}$-set then the $\mathscr{E}$-maps $f: A \rightarrow B$ are precisely the *continuous maps $f: A \rightarrow^{*} Y$ such that $f(A) \subseteq B$.

The most obvious examplès of $\mathscr{E}$-maps $f: A \rightarrow B$ are obtained by restricting to $A$ some internal *continuous map $g$ which is defined on an internal set $A^{\prime} \supseteq A$ and maps $A$ into $B$. It is natural to conjecture that all $\mathscr{E}$-maps arise in this way. However, Ward Henson using techniques he has developed in [7] has shown that even if $A=\mu(0)$, the monad of 0 in ${ }^{*} \boldsymbol{R}$, there are $\mathscr{E}$-maps $f: \mu(0) \rightarrow{ }^{*} \boldsymbol{R}$ which cannot be extended to any internal set containing $\mu(0)$.

The usual basic definitions and results of homotopy thẹory (see, e.g. [8] or [14]) carry over unchanged to the category $\mathscr{E}$ as follows.

I. 2. Defrntrton. If $A \subseteq B$ and $C \subseteq D$ are all $\mathscr{E}$-sets then an $\mathscr{E}$-map $f:(B, A) \rightarrow(D, C)$ is an $\mathscr{E}$-map $f: B \rightarrow D$ such that $f(A) \subseteq C$.

If $A \subseteq * X$ is an $\mathscr{E}$-set then so is $A \times{ }^{*} I \subseteq *(X \times I)$ where $I=[0,1]$. Thus, we can make the following definitions.

1. 3. Definitron. Suppose $A \subseteq B$ and $C \subseteq D$ are all $\mathscr{E}$-sets, and $f:(B, A) \rightarrow(D, C)$ and $g:(B, A) \rightarrow(D, C)$ are $\mathscr{E}$-maps. A homotopy $F$ from $f$ to $g$ is an $\mathscr{E}$-map $F:(B \times * I, A \times * I) \rightarrow(D, C)$ such that:

(i) for every $b \in B, F(b, 0)=f(b)$,

(ii) for every $b \in B, F(b, 1)=g(b)$. 
If such a homotopy exists we say $f$ is homotopic to $g$ and write $f \sim g:(B, A) \rightarrow(D, C)$, or sometimes, simply, $f \sim g$.

The proofs of the following propositions are exactly the same as the standard proofs with the observation that the usual constructions (e.g. $G(x, t)=F(x, 1-t)$ ) all work within the category $\mathscr{E}$.

I. 4. Proposition. $\sim$ is an equivalence relation.

I. 5. Proposition. If $f \sim f^{\prime}:(A, B) \rightarrow(C, D)$ and $g \sim g^{\prime}:(C, D) \rightarrow(E, F)$ then $g f \sim g^{\prime} f^{\prime}:(A, B) \rightarrow(E, F)$.

I. 6. Definition. Two $\mathscr{E}$-sets $A$ and $B$ are said to have the same homotopy type provided there are $\mathscr{E}$-maps $f: A \rightarrow B$ and $g: B \rightarrow A$ such that $g f \sim I_{A}$ and $f g \sim I_{B}$ (where $I_{A}$ and $I_{B}$ denote the identity maps on $A$ and $B$ respectively.)

I. 7. Propositron. Having the same homotopy type is an equivalence relation.

I. 8. Definition. Suppose $A$ is an $\mathscr{E}$-set and $a \in A$. Let $\Omega(A, a)$ be the set of all $\mathscr{E}$-maps $\omega:{ }^{*} I \rightarrow A$ such that $\omega(0)=\omega(1)=a$. Notice, the elements of $\Omega(A, a)$ are all internal * continuous maps but the set $\Omega(A, a)$ itself is, in general, external.

If $u, v \in \Omega(A, a)$ we define $u * v \in \Omega(A, a)$ as usual by

$$
(u * v)(t)=\left\{\begin{array}{llc}
u(2 t) & \text { if } & 0 \leqslant t \leqslant \frac{1}{2}, \\
v(2 t-1) & \text { if } & \frac{1}{2} \leqslant t \leqslant 1 .
\end{array}\right.
$$

We write $u \sim v$ if $u \sim v:\left({ }^{*} I,\{0,1\}\right) \rightarrow(A, a)$ and let $[u]=\{v \in \Omega(A, a) \mid u \sim v\}$.

Finally the fundamental group of $A$ based at $a$, denoted $\pi_{1}(A, a)$ is defined by,

$$
\begin{gathered}
\pi_{1}(A, a)=\{[u] \mid u \in \Omega(A, a)\}, \\
{[u] *[v]=[u * v] .}
\end{gathered}
$$

I. 9. Proposttion. $\pi_{1}(A, a)$ with the operation $*$ is a group.

I. 10. Proposition. Suppose $a_{0}, a_{1} \in A$ and there is a path from $a_{0}$ to $a_{1}$ in $A$ then $\pi_{1}\left(A, a_{0}\right)$ is isomorphic to $\pi_{1}\left(A, a_{1}\right)$.

Notice that when the $\mathscr{E}$-sets in the preceding definitions are internal, these definitions are the usual ones (or, more precisely, the nonstandard extensions of the usual ones).

I. 11. Definttron. An $\mathscr{E}$-map $f: A \rightarrow B$ is said to be strong if there is a standard set $S$ such that $A \subseteq{ }^{*} S$ and a standard continuous function $F$ defined on $S$ such that $f=\left.{ }^{*} F\right|_{A}$. Two strong maps are said to be strongly homotopic if there is a strong homotopy between them. Two $\mathscr{E}$-sets have the same strong homotopy type if the maps and homotopies of Definition I.6 are all strong.

Throughout the remainder of this paper unless explicitly stated to the contrary all sets and mappings will be in the category $\mathscr{E}$. We close this section with one of the examples which motivates this work.
T. 12. Example. Let $C=\left\{(x, \sin (1 / x)) \in \boldsymbol{R}^{2} \mid 0<x \leqslant 1\right\} \cup(0 \times[-1,1])$.

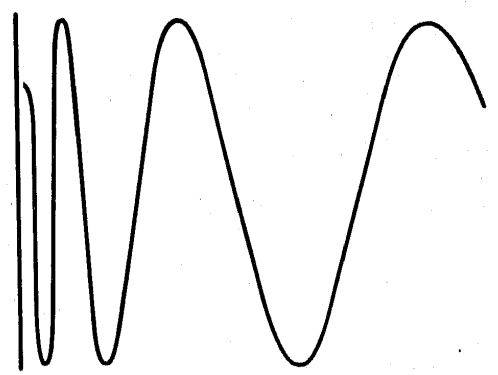

Let $A=\left\{x \in *^{*} R^{2} \mid \mathrm{S}:(x) \in C\right\}$.

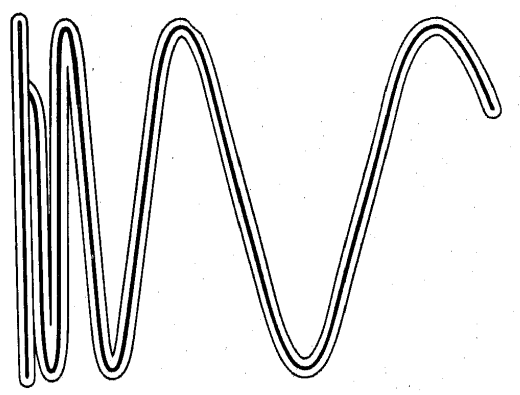

Then we can see that $A$ and $* I$ have the same homotopy type by considering the maps $f:{ }^{*} I \rightarrow A$ and $g: A \rightarrow{ }^{*} I$ defined by

$$
\begin{gathered}
f(x)=\left\{\begin{array}{llc}
\left(x, \sin \frac{1}{x}\right) & \text { if } & \frac{1}{2 \pi \nu} \leqslant x \leqslant 1, \\
(x, 0) & \text { if } & 0 \leqslant x \leqslant \frac{1}{2 \pi \nu},
\end{array}\right. \\
g(x, y)=\left\{\begin{array}{llc}
0 & \text { if } & x \leqslant 0, \\
x & \text { if } & 0 \leqslant x \leqslant 1, \\
1 & \text { if } & 1 \leqslant x .
\end{array} .\right.
\end{gathered}
$$

where $v$ is any fixed infinite positive integer. Both maps are clearly $\mathscr{E}$-maps, $g f=I^{*}$ and the following homotopy $F$ shows $f g \sim I_{A}$

$$
F(x, y, t)=(1-t)(x, y)+t(f g(x, y)) \text {. }
$$

Thus $A$ has the same homotopy type as * $I$ and, hence, by Proposition I.7 and the remarks following Proposition I.10, as a point. Notice, however, that $C$ does not have the same homotopy type as a point and that $A$ and a point do not have the same strong homotopy type. 
II. The envelope functor. In this section we will make extensive use of the following two results which can be found, for example, in [5].

II. 1. Theorem. Suppose $X$ is a (metric) space. Then there is a normed linear space $Y$ and an isometric embedding $e: X \rightarrow Y$ such that $e(X)$ is a closed subset of its convex hull $H(X, Y)$ in $Y[5, X I I I .5 .2]$.

II. 2. Theorem. Suppose $A$ is a closed subspace of a (metric) space $Y$, and $L$ is a normed linear space and $f: A \rightarrow L$. Then there is an extension $F: Y \rightarrow L$ of $f$ such that $F(Y)$ is contained in the convex hull of $f(A)$ in $L$ [5, IX. 6.1].

As in [15] and [16] we define the monad of an arbitrary point $x \in{ }^{*} X$ as follows.

II. 3. Definition.

$\mu(x)=\left\{y \in{ }^{*} X \mid\right.$ for every standard, continuous

$$
\sigma: X \rightarrow(0,1], * d(x, y)<* \sigma(x)\} .
$$

Recall from [15] and [16] that the monads form a partition of $* X$ and are preserved by standard continuous functions. In fact, a standard function $f: X \rightarrow Y$ is continuous if and only if for every $x \in * X, * f(\mu(x)) \subseteq \mu(f(x))$. Notice, in particular, that if $x$ is near-standard then $\mu(x)=\mu(\mathrm{S}:(x))$ and thus for standard points the two definitions of $\mu(x)$ agree. The proofs of the following lemmas are completely straightforward.

II. 4. LEMMA. Suppose $F$ is a standard closed subset of $X, U$ is a standard open subset of $X, F \subseteq U$ and $x \in *^{*} F$. Then $\mu(x) \subseteq{ }^{*} U$.

II. 5. Lemma. Suppose $F$ is a standard closed subset of $X$. Let $\mu(x, F)$ denote the monad of $x$ in the space $* F$ and let $\mu(x, X)$ denote the monad of $x$ in the space ${ }^{*} X$. Then $\mu(x, F)=\mu(x, X) \cap * F$.

We can now define the envelope of a (metric) space as follows.

II. 6. Definition. Suppose $X$ is a (metric) space. By Theorem II. 1 we may assume $X$ is a subset of a normed linear space $S$ and that $X$ is a closed subset of its convex hull $H(X, S)$ in $S$. The envelope of $X$ in $S$, denoted $\mathscr{E}(X, S)$ is defined by,

$$
\mathscr{E}(X, S)=\{y \in * H(X, S) \mid \mu(y) \cap * X \neq \emptyset\}
$$

where $\mu(y)$ denotes the monad of $y$ in $* H(X, S)$.

We have immediately the following alternative description of $\mathscr{E}(X, S)$.

$$
\text { II. 7. Proposition. } \mathscr{E}(X, S)=\underset{X \subseteq V \subseteq H(X, S), \gamma_{\text {open }}}{ } * V
$$$$
\text { Proof. Let } E=\bigcap^{*} * V \text {. }
$$

(i) By Lemma ח. $4 \mathscr{E}(X, S) \subseteq E$.

(ii) Now suppose $y \notin \mathscr{E}(X, S)$.

We claim there is a standard continuous function $f: X \rightarrow(0,1]$ such that for every $x \in * X, * d(x, y)>{ }^{*} f(x)$. If no such $f$ exists then the relation $R(f, x)=x \in{ }^{*} X$ and $* d(x, y) \leqslant * f(x)$ is finitely satisfiable for standard continuous functions, $f: X \rightarrow(0,1]$. Then since ${ }^{*} \mathscr{M}$ is $x^{+}$saturated there is some $x \in * X$ such that
$* d(x, y) \leqslant{ }^{*} f(x)$ for all standard continuous $f: X \rightarrow(0,1]$. Thus, $y \in \mu(x) \subseteq \mathscr{E}(X, S)$. Therefore, the claim is true.

Let $f$ be such a function and let $V=\{z \in H(X, S) \mid$ for some $x \in X, d(x, z)<f(x)\}$. $V$ is a standard open set containing $X$ and $y \notin * V$. Therefore $y \notin E$ which completes the proof.

The set $\mathscr{O}(X, S)$ is locally convex in the following sense.

II. 8. Lemma. Suppose $x \in * X$ and $\left(x_{1}, x_{2}, \ldots, x_{v}\right)$ is an internal *finite sequence of elements of $\mu(x)$. Suppose $\left(\lambda_{1}, \lambda_{2}, \ldots, \lambda_{v}\right)$ is an internal ${ }^{*}$ finite sequence in ${ }^{*} I$ such that $\sum \lambda_{i}=1$. Then $\sum \lambda_{i} x_{i} \in \mu(x) \subseteq \mathscr{E}(X, S)$.

Prool.

$$
\begin{aligned}
*\left\|\left(\sum \lambda_{i} x_{i}\right)-x\right\| & =*\left\|\left(\sum \lambda_{i} x_{i}\right)-\left(\sum \lambda_{i} x\right)\right\| \\
& =*\left\|\sum \lambda_{i}\left(x_{i}-x\right)\right\| \leqslant \sum \lambda_{i}\left\|x_{i}-x\right\| .
\end{aligned}
$$

Now, for any standard continuous function $f: H(X, S) \rightarrow(0,1]$ we have for each $i$, $*\left\|x_{i}-x\right\|<{ }^{*} f(x)$ since $x_{i} \in \mu(x)$. Hence, $\sum \lambda_{i}\left\|x_{i}-x\right\|<\sum \lambda_{i}{ }^{*} f(x)={ }^{*} f(x)$. Thus $\sum \lambda_{i} x_{i} \in \mu(x) \subseteq \mathscr{E}(X, S)$.

Next, we use Theorem II. 2 to define the envelope $\mathscr{E}(f)$ of a map $f: X \rightarrow Y$.

II. 9. Proposition. Suppose $X$ and $Y$ are embedded in the normed linear spaces $S$ and $T$ respectively as closed subsets of their convex hulls. Suppose $f: X \rightarrow Y$ is a continuous map. Then there is a strong $\mathscr{E}-\operatorname{map} F: \mathscr{E}(X, S) \rightarrow \mathscr{E}(Y, T)$ extending $f$. Furthermore, if $F_{1}$ and $F_{2}$ are any two such maps then they are strongly homotopic.

proof. By Theorem Ir. $2 f$ can be extended to a map $g: H(X, S) \rightarrow H(Y, T)$. Since $g$ is standard and continuous for every $x \in{ }^{*} X,{ }^{*} g(\mu(x)) \subseteq \mu\left({ }^{*} g(x)\right)$. So, ${ }^{*} g(\mathscr{E}(X, S)) \subseteq \mathscr{E}(X, T)$. Hence, we may define $F=\left.g\right|_{\mathscr{\&}(X, S)}$ which is the desired map.

Now, suppose $F_{1}$ and $F_{2}$ are two strong $\mathscr{E}$-maps extending $f$. Then $F_{1}$ and $F_{2}$ are restrictions of standard continuous maps $\bar{F}_{1}: A_{1} \rightarrow T, \bar{F}_{2}: A_{2} \rightarrow T$ respectively. Let $A=A_{1} \cap A_{2}$ and define $G: A \times I \rightarrow T$ by

$$
G(x, t)=t \bar{F}_{1}(x)+(1-t) \bar{F}_{2}(x) .
$$

Now, if $x \in \mathscr{E}(X, S)$ there is an $x_{0} \in * X$ such that $x \in \mu\left(x_{0}\right)$. Since $\bar{F}_{1}$ and $\bar{F}_{2}$ are standard and continuous, $\bar{F}_{1}(x) \in \mu\left({ }^{*} f\left(x_{0}\right)\right)$ and $\bar{F}_{2}(x) \subseteq \mu\left(* f\left(x_{0}\right)\right)$. So, by Lemma II. 8 , $G(x, t) \in \mu\left({ }^{*} f\left(x_{0}\right)\right) \subseteq \mathscr{E}(Y, T)$. Thus, $G(\mathscr{E}(X, S)) \subseteq \mathscr{E}(Y, T)$ and $\left.G\right|_{\mathscr{E}(X, T)}$ is the desired strong homotopy.

IT. 10. Corolxary. Suppose $X$ is embedded in the normed linear spaces $S$ and $T$, in each case as a closed subset of its convex hull. Then $\mathscr{E}(X, S)$ and $\mathscr{E}(X, T)$ have the same strong homotopy type.

Proof. By Proposition II, 9 the identity map $I: X \rightarrow X$ induces strong $\mathscr{E}$-maps $f: \mathscr{E}(X, S) \rightarrow \mathscr{E}(X, T)$ and $g: \mathscr{E}(X, T) \rightarrow \mathscr{E}(X, S)$. Now, $f g: \mathscr{E}(X, T) \rightarrow \mathscr{E}(X, T)$ is a strong $\mathscr{E}$-map extending the identity map $I: X \rightarrow X$, but so is the identity map on $\mathscr{E}(X, T)$ So by Proposition II. $9 \mathrm{fg}$ is strongly homotopic to the identity map on $\mathscr{E}(X, T)$. Similarly, $g f$ is strongly homotopic to the identity map on $\mathscr{E}(X, S)$. on $\mathscr{E}(X, T)$. Similat 
In view of Corollary II. 10 we write $\mathscr{E}(X)$ instead of $\mathscr{E}(X, S)$ and note that $\mathscr{E}(X)$ is well-defined up to strong homotopy type. Similarly, if $f: X \rightarrow Y$ we write $\mathscr{E}(f): \mathscr{E}(X) \rightarrow \mathscr{E}(Y)$ which is well defined by Proposition II. 9 up to strong homotopy.

In general, $X$ and $\mathscr{E}(X)$ do not have the same strong homotopy type or even the same homotopy type (see Example I. 12). However, for absolute neighborhood retracts we have the following.

II. 11. Proposition. Suppose $X$ is an absolute neighborhood retract. (ANR) (see, for example, [14]). Then, $X$ and $\mathscr{E}(X)$ have the same strong homotopy type.

Proof. Let $X$ be embedded as a closed subset of its convex hull $\operatorname{Fr}(X, S)$ in a normed linear space $S$. Since $X$ is an ANR there is an open neighborhood $U$ of $X$ in $H(X, S)$ and a retraction $R: U \rightarrow X$. By Proposition II. $7 \mathscr{E}(X, S) \subseteq{ }^{*} U$ so $R$ restricts to a strong $\mathscr{E}$-map $r: \mathscr{E}(X, S) \rightarrow{ }^{*} X$. Let $i: * X \rightarrow \mathscr{E}(X, S)$ be the inclusion map. Since $r$ is a retraction $r i=I_{* X}$. Define $\bar{F}: U \times I \rightarrow S$ by

$$
\bar{F}(x, t)=t R(x)+(1-t) x .
$$

If $x \in \mathscr{E}(X, S)$ then for some $x_{0} \in{ }^{*} X, x \in \mu\left(x_{0}\right)$. Since $R$ is a standard and continuous $* R(x) \in \mu\left({ }^{*} R\left(x_{0}\right)\right)=\mu\left(x_{0}\right)$. Hence, by Lemma II. 8 for all $t \in * I$ $* \bar{F}(x, t) \in \mu\left(x_{0}\right) \subseteq \mathscr{E}(X, S)$. Hence $\bar{F}$ restricts to an $\mathscr{E}$-map $F: \mathscr{E}(X, S) \times{ }^{*} I \rightarrow \mathscr{E}(X, S)$ which is clearly a strong homotopy from $i R$ to the identity on $\mathscr{E}(X, S)$.

One of the valuable properties of Čech Homology is that $H_{0}(X)$ measures the number of components of $X$ while in singular homology $H_{0}(X)$ measures the number of path components of $X$. The following theorem shows that interposing the envelope functor also has the effect of converting connectedness into path connectedness.

II. 12. THEOREM. (i) $\mathscr{E}(X)$ is path-connected if and only if $X$ is connected.

(ii) Moreover, if $X$ is connected then for every internal set $A \subseteq \mathscr{E}(x)$ there is an internal, path-connected, locally convex set $A^{\prime}$ such that $A \subseteq A^{\prime} \subseteq \mathscr{E}(X)$.

Proof. First, we show if $X$ is not connected then $\mathscr{E}(X)$ is not path connected. Then we prove (ii) which implies the other half of (i).

(i) If $X$ is not connected there are two nonempty disjoint closed sets $G$ and $H$ such that $X=G \cup H$. Let $X$ be embedded as a closed subset of its convex hull $H(X, S)$ in a normed linear space $S$. Then $G$ and $H$ are closed subsets of $H(X, S)$. Since $H(X, S)$ is a metric space it is normal and there are disjoint open subsets $U$ and $V$ of $H(X, S)$ such that $G \subseteq U$ and $H \subseteq V$. By Proposition II. 7 $\mathscr{E}(X, S) \subseteq \subseteq^{*} U \cup^{*} V$.

Now choose $a \in G$ and $b \in H$. If $\omega:{ }^{*} I \rightarrow \mathscr{E}(X, S)$ is a path from $a$ to $b$ then $\omega^{-1}(* U)$ and $\omega^{-1}(* V)$ are nonempty disjoint *open subsets of * $I$ such that $\omega^{-1}\left({ }^{*} U\right) \cup \omega^{-1}(* V)={ }^{*} I$ which is impossible since $* I$ is $*$ connected.

(ii) We may assume $* X \subseteq A$. Since $A$ and $* X$ are internal we can define a (discontinuous) map $\tau: A \rightarrow^{*} X$ such that $* d(a, \tau(a))<2 \inf \left\{* d(a, x) \mid x \in{ }^{*} X\right\}$ and a map $\sigma:{ }^{*} X \rightarrow{ }^{*} I$ such that $\sigma(x)=\sup \{* d(x, a) \mid \tau(a)=x\}$. Since $* \mathscr{M}$ is an enlargement there is an internal, *continuous function $\alpha:{ }^{*} H(X, S) \rightarrow(0,1]$ such that for every $x \in * H(X, S)$ and every standard continuous function $f: H(X, S) \rightarrow(0,1], \alpha(x)<* f(x)$. Let $A^{\prime}$ be given by

$$
\begin{aligned}
A^{\prime} & =\{y \in * H(X, S) \mid \text { for some } x \in * X, * d(x, y)<\sigma(x)+\alpha(x)\} \\
& =\bigcup_{x \in * X}\{y \in * H(X, S) \mid * d(x, y)<\sigma(x)+\alpha(x)\} .
\end{aligned}
$$

By the choice of $\sigma$ and $\alpha, A \subseteq A^{\prime} \subseteq \mathscr{E}(X, S)$. Since for each $x \in * X$, the set $\left\{y \in{ }^{*} H(X, S) \mid{ }^{*} d(x, y)<\sigma(x)+\alpha(x)\right\}$ is *convex, $A^{\prime}$ is clearly locally convex. . Furthermore, since each of these sets intersects the *connected set ${ }^{*} X, A^{\prime}$ is also *connected and, hence, path-connected.

We close this section by remarking that the exact relationship between the techniques developed here and Borsuk's "Theory of Shape" ([1], [2]) is unknown. In particular, the relationship between the homotopy type of $\mathscr{E}(X)$ and the shape of $X$ is unknown.

III. Covering spaces. For reasonably nice spaces $B$ (connected, locally pathwise connected, and semi-locally 1 -connected) the theory of covering spaces $p: E \rightarrow B$ is particularly elegant. Such covering spaces are completely classified by subgroups $\left(p_{*}\left(\pi_{1}(E, e)\right)\right)$ of the fundamental group of $B$. For more general spaces, however this classification breaks down. For example, the Warsaw circle has a trivial fundamental group but essentially the same covering spaces as the circle. In [6] Fox obtained a classification of covering spaces $p: E \rightarrow B$ for more general spaces $B$ using shape theory and particularly his notion of the "fundamental trope" of $B$. The purpose of this section is to obtain a classification of such covering spaces in terms of subgroups of $\pi_{1}(\mathscr{E}(B))$.

There are a number of possible definitions of the notion of "covering space." For reasonably nice spaces these definitions coincide. However, for more general spaces these definitions do not coincide. We will be interested here in three such definitions. The first and strongest is due essentially to Fox [6] and is the definition we will eventually use.

Irr. 1. Definition. Suppose $E$ and $B$ are connected spaces and $p: E \rightarrow B$ is surjection. Suppose $b \in B$ and $F=p^{-1}(b)$ is discrete. An overlay structure for $p: E \rightarrow B$ is an open covering $\mathfrak{H}=\left\{U_{\alpha}\right\}$ of $B$ together with homeomorphisms $\varphi_{\alpha}: U_{\alpha} \times F \rightarrow p^{-1}\left(U_{\alpha}\right)$ such that

(i) For each $\alpha$ the diagram

$$
U_{\alpha} \times F \stackrel{p_{a}}{\longrightarrow} p^{-1}\left(U_{\alpha}\right)
$$

commutes (where $p_{1}(x, t)=x$ ).

(ii) For each $\alpha, \beta$ there is a homeomorphism $g_{\alpha \beta}: F \rightarrow F$ such that for every $x \in U_{\alpha} \cap U_{\beta}$ and $t \in F$

$$
\varphi_{\alpha}(x, t)=\varphi_{\beta}\left(x, g_{\alpha \beta}(t)\right)
$$

(Notice $g_{\alpha \beta}$ does not depend on the point $x \in U_{\alpha} \cap U_{\beta}$.)

4 - Fundamenta Mathematicae XCVrrr 
An overlay is a map $p: E \rightarrow B$ as above with a given overlay structure.

III. 2. Defintrion. Suppose $p: E \rightarrow B$ is an overlay with a given overlay struc- ture $\left\langle\left\{U_{\alpha}\right\},\left\{\varphi_{\alpha}\right\}\right\rangle$. A second overlay structure $\left\langle\left\{V_{\beta}\right\},\left\{\psi_{\beta}\right\}\right\rangle$ is said to refine $\left\langle\left\{U_{\alpha}\right\},\left\{\varphi_{\alpha}\right\}\right\rangle$ provided

(i) $\left\{V_{\beta}\right\}$ refines $\left\{U_{\alpha}\right\}$.

(ii) For every $\alpha, \beta$ such that $V_{\beta} \subseteq U_{\alpha}$ there is a homeomorphism $\sigma_{\alpha \beta}: F \rightarrow F$ such - that for every $x \in V_{\beta}, t \in F, \varphi_{\alpha}(x, t)=\psi_{\beta}\left(x, \sigma_{\alpha \beta}(t)\right)$.

Two overlay structures are said to be equivalent if there is an overlay structure which refines both of them.

The other two notions of covering space we are concerned with are the usual one and that of a unique path lifting fibration. These definitions are given below for convenience.

III. 3. Definition. A covering space consists of two connected spaces, $E$ and $B$ and a map $p: E \rightarrow B$ such that for each $x \in B$ there is an open set $U$ containing $x$ such that $p^{-1}(U)$ is a disjoint union of open sets $\left\{V_{x}\right\}$ and for each $x,\left.p\right|_{V_{x}}: V_{x} \rightarrow U$ is a homeomorphism.

III. 4. Definition. A unique path lifting fibration (UPL-fibration) is a Hurewicz fiber space [14] $p: E \rightarrow B$ such that

(i) $E$ is connected.

(ii) For each path $\omega: I \rightarrow B$ and each $e \in p^{-1}(\omega(0))$ there is a unique path $\bar{\omega}: I \rightarrow E$ such that $p \bar{\omega}=\omega$ and $\bar{\omega}(0)=e$.

Clearly, every overlay is a covering space. It is well-known that every covering space is a UPL-fibration. The following example is a UPL-fibration which is not a covering space. For examples of covering spaces with no overlay structures, or with two inequivalent overlay structures see [12].

III. 5. EXAMPLE. Let $S$ denote the unit circle in the complex plane and let $S^{\omega}=S \times S \times S \times \ldots$ with the Tychonoff topology. Let $R$ denote the real line and $R^{\omega}=R \times R \times R \times \ldots$ with the Tychonoff topology. Define $p: R^{\omega} \rightarrow S^{\omega}$ by $p\left(t_{1}, t_{2}, \ldots\right)$ $=\left(e^{2 \pi i t_{1}}, e^{2 \pi i t_{2}}, \ldots\right)$. It is easy to verify that $p: R^{\omega} \rightarrow S^{\omega}$ is a UPL-fibration. However, it is also easy to see using, for example, $\left[14\right.$, II. 5.11] that $p: R^{\omega} \rightarrow S^{\omega}$ is not a covering space.

Most of the useful properties of covering spaces result entirely from the unique path lifting property. Thus, one would really like to obtain a classification theorem for UPL-fibrations. Unfortunately, both the present work and [6] is limited to overlays.

Our approach to the problem of classifying overlays $p: E \rightarrow B$ is to work instead with covering spaces $\tilde{p}: \tilde{E} \rightarrow \mathscr{E}(B)$ in the category $\mathscr{E}$. Since $\mathscr{E}(B)$ is locally convex $\tilde{p}: \tilde{E} \rightarrow \mathscr{E}(B)$ will necessarily be an overlay, Our work is divided into three parts. First, starting with a standard overlay $p: E \rightarrow B$ we show using essentially the same technique as Fox [6] that there is an extension $\tilde{p}: \tilde{E} \rightarrow \mathscr{E}(B)$ of $p: E \rightarrow B$. Then we show how certain covering spaces $q: F \rightarrow \mathscr{E}(B)$ give rise to standard overlays $p: E \rightarrow B$. Finally, we show that in the category $\mathscr{E}$ the theory of covering spaces $q: F \rightarrow \mathscr{E}(B)$ is particularly nice. Before defining covering spaces in the category $\mathscr{E}$ we consider an example.
III. 6. ExAMPLE. Let $S$ denote the unit circle in the complex plane and let $F \subseteq * R$ be the set of all finite nonstandard reals. Let $p: F \rightarrow{ }^{*} S$ be given by $p(t)=e^{2 \pi i t}$. Clearly $p: F \rightarrow{ }^{*} S$ satisfies the definition of an overlay (Definition III. 1) and is an $\mathscr{E}$-map. However, it does not have the path lifting property. In fact, if $\lambda$ is any infinite real the path $\omega(t)=e^{2 \pi i \lambda t}$ has no lifting. Hence, we need a more careful definition of covering space in the category $\mathscr{E}$.

III. 7. Definition. An $\mathscr{E}$-covering space consists of two $\mathscr{E}$-sets, $E$ and $B$, and an $\mathscr{E}$-map $p: E \rightarrow B$ such that

(i) $E$ is path connected.

(ii) For every internal set $A \subseteq B, E_{A}=p^{-1}(A)$ is internal and $\left.p\right|_{E_{A}}: E_{A} \rightarrow A$ is a *covering space (except that $E_{\boldsymbol{A}}$ is allowed to be disconnected).

The following lemma due to Kuratowski (see [9; Chapter II, §21, XI, Theorem 2]) and theorem due to Fox [6] allow an overlay $p: E \rightarrow B$ to be extended to an $\mathscr{E}$-covering space $\tilde{p}: \tilde{E} \rightarrow \mathscr{E}(B)$.

III. 8. LemMa. Suppose $B$ is a closed subset of a (metric) space $X$ and $\mathscr{U}=\left\{U_{\alpha}\right\}$ is a covering of $B$ by open subsets of $B$. Then there are open subsets $\left\{V_{\alpha}\right\}$ of $X$ such that

(i) $U_{\alpha}=B \cap V_{\alpha}$.

(ii) For each $\alpha_{1}, \alpha_{2}, \ldots, \alpha_{k}$,

$$
V_{\alpha_{1}} \cap V_{\alpha_{2}} \cap \ldots \cap \dot{V}_{\alpha_{k}} \neq \emptyset \leftrightarrow U_{\alpha_{1}} \cap U_{\alpha_{2}} \cap \ldots \cap U_{\alpha_{k}} \neq \emptyset .
$$

Proof. [9].

III. 9. THEOREM (Fox [6]). Suppose $p: E \rightarrow B$ is an overlay and $B$ is a closed subset of a metric space $X$. Then there is an open subset $U$ of $X$ containing $B$ and an overlay $\hat{p}: \hat{H} \rightarrow U$ such that $\hat{p}^{-1}(B)=E$ and $\left.\hat{p}\right|_{E}=p$.

Proof. Suppose $\left\langle\left\{U_{\alpha}\right\},\left\{\varphi_{\alpha}\right\}\right\rangle$ is an overlay structure for $p: E \rightarrow B$. Let $g_{\alpha \beta}: F \rightarrow F$ be the homeomorphisms provided by Definition III. 1. Let $\left\{V_{\alpha}\right\}$ be a collection of open subsets of $X$ provided by Lemma III. 8. Let $U=U V_{\alpha}$ and define $H$ to be the disjoint union $U\left(V_{\alpha} \times F\right)$. If $(x, t) \in V_{\alpha} \times F$ and $(x, s) \in V_{\beta} \times F$ we write $(x, t) \sim(x, s)$ provided $s=g_{\alpha \beta}^{\alpha}(t)$. This is an equivalence relation since $V_{\alpha} \cap V_{\beta} \cap V_{\gamma} \neq \varnothing$ implies $U_{\alpha} \cap U_{\beta} \cap U_{\gamma} \neq \varnothing$ which implies $g_{\beta \gamma} g_{\alpha \beta}=g_{\alpha \gamma}$. Let $\hat{H}=H / \sim$ and $\hat{p}: \hat{H} \rightarrow U$ be the obvious map. This is easily seen to be the soughtfor extension of $p: E \rightarrow B$.

III. 10. Corollary. Suppose $p: E \rightarrow B$ is an overlay. Then there is an $\mathscr{E}$-covering space $\tilde{p}: \tilde{E} \rightarrow \mathscr{E}(B)$ such that $\tilde{p}^{-1}\left({ }^{*} B\right)=* E$ and $\left.\tilde{p}\right|_{* E}=p$.

Proof. Immediate from Theorem III. 9.

The following proposition shows that such extensions exist only for overlays and that two such extensions are equivalent as $\mathscr{E}$-covering spaces if and only if the given overlay structures are equivalent.

III. 11. Propositron. (i) Suppose $p: E \rightarrow B$ is a (standard) covering space and $\tilde{p}: \tilde{E} \rightarrow \mathscr{E}(B)$ is a $\mathbb{E}$-covering space extending $p: E \rightarrow B$. Then $p: E \rightarrow B$ is an overlay.

(ii) Suppose $\left\langle\left\{U_{\alpha}\right\},\left\{\varphi_{\alpha}\right\}\right\rangle$ and $\left\langle\left\{V_{\beta}\right\},\left\{\psi_{\beta}\right\}\right\rangle$ are two overlay structures on $p: E \rightarrow B$ and $\tilde{p}: \tilde{E} \rightarrow \mathscr{E}(B)$ and $\hat{p}: \hat{E} \rightarrow \mathscr{E}(B)$ are extensions constructed from $\left\langle\left\{U_{\alpha}\right\},\left\{\varphi_{\alpha}\right\}\right\rangle$ 
and $\left\langle\left\{V_{\beta}\right\},\left\{\psi_{\beta}\right\}\right\rangle$ respectively as in III. 9 and III. 10. Then $\tilde{p}: \tilde{E} \rightarrow \mathscr{E}(B)$ and $\hat{p}: \hat{E} \rightarrow \mathscr{E}(B)$ are equivalent as $\mathscr{E}$-covering spaces if and only if $\left\langle\left\{U_{\alpha}\right\},\left\{\varphi_{\alpha}\right\}\right\rangle$ and $\left\langle\left\{V_{\beta}\right\},\left\{\psi_{\beta}\right\}\right\rangle$ are equivalent overlay structures.

Proof. (i) Since $* \mathscr{M}$ is an enlargement there is a *continuous function $\sigma:{ }^{*} B \rightarrow^{*}(0,1]$ such that for every standard continuous function $f: B \rightarrow(0,1]$ for all $x \in * B, \sigma(b)<* f(b)$. Let $V=\left\{x \in * X \mid \exists b^{*} d(b, x)<\sigma(b)\right\} . V$ is internal, so $\tilde{E}_{Y}=\tilde{p}^{-1}(V)$ is also internal and $\left.\tilde{p}\right|_{\tilde{E}_{V}}: \tilde{E}_{V} \rightarrow V$ is a ${ }^{*}$ covering space. For each $b \in^{*} B$, let $V_{b}=\{x \in * X \mid * d(b, x)<\sigma(b)\}$. Each $V_{b}$ is *convex and, hence, $\tilde{p}^{-1}\left(V_{b}\right)$ is a disjoint union of *open sets $W_{b i}$ such that $\left.\tilde{p}\right|_{W_{b} t}: W_{b i} \rightarrow V_{b}$ is a *homeomorphism for each $i$. If $V_{b} \cap V_{a} \neq \varnothing$ it is * convex and, hence, each $W_{b l}$ intersects a unique $W_{a j}$. Thus, $\left.\tilde{p}\right|_{\tilde{E}_{V}} \rightarrow V$ has a *overlay structure which restricts to a $*_{\text {overlay structure on }}$ ${ }^{*} p$ : ${ }^{*} E \rightarrow B$ and, since ${ }^{*} \mathscr{M}$ is an elementary extension of $\mathscr{M}, p: E \rightarrow B$ has an overlay structure.

(ii) $(\rightarrow)$ Since $B$ is paracompact we may assume $\left\{U_{\alpha}\right\}$ and $\left\{V_{\beta}\right\}$ are locally finite. Let $V, V_{b}, \tilde{E}_{V}$, and $\hat{E}_{V}$ be defined as above. The covering $\left\{V_{b}\right\}$ of $* B$ refines both $\left\{U_{\alpha}\right\}$ and $\left\{V_{\beta}\right\}$ by [15, Theorem 2.12]. The *overlay structure defined above on ${ }^{*} p$ : ${ }^{*} E \rightarrow{ }^{*} B$ using $\left\{V_{b} \cap{ }^{*} B\right\}$ is easily seen to be a *refinement of both ${ }^{*}\left\langle\left\{U_{\alpha}\right\},\left\{\varphi_{\alpha}\right\}\right\rangle$ and $*\left\langle\left\{V_{\beta}\right\},\left\{\psi_{\beta}\right\}\right\rangle$. Thus, they have a common *refinement and, hence $\left\langle\left\{U_{\alpha}\right\},\left\{\varphi_{\alpha}\right\}\right\rangle$ and $\left\langle\left\{V_{\beta}\right\},\left\{\psi_{\beta}\right\}\right\rangle$ have a common refinement.

$(\leftarrow)$ This is immediate from the straightforward observation that the implication is true if $\left\langle\left\{V_{\beta}\right\},\left\{\psi_{\beta}\right\}\right\rangle$ refines $\left\langle\left\{U_{\alpha}\right\},\left\{\varphi_{\alpha}\right\}\right\rangle$.

Next, suppose $p: E \rightarrow B$ and $p^{\prime}: E^{\prime} \rightarrow B^{\prime}$ are two overlays and that $(f, f)$ is a covering transformation (i.e. $f: B \rightarrow B^{\prime}, \bar{f}: E \rightarrow E^{\prime}$ and $p^{\prime} f=f_{p}$ ). In view of [12], $(f, \bar{f})$ might not have an extension to an $\mathscr{E}$-covering transformation $(\mathscr{E}(f), f)$ from $\tilde{p}: \tilde{E} \rightarrow \mathscr{E}(B)$ to $\tilde{p}^{\prime}: \tilde{E}^{\prime} \rightarrow \mathscr{E}\left(B^{\prime}\right)$. Thus we make the following definition.

III. 12. Defintrion. Suppose $p: E \rightarrow B$ is an overlay with a given overlay structure $\left\langle\left\{U_{\alpha}\right\},\left\{\varphi_{\alpha}\right\}\right\rangle$ and fiber $F$, and $p^{\prime}: E^{\prime} \rightarrow B^{\prime}$ is an overlay with a given overlay structure $\left\langle\left\{V_{\beta}\right\},\left\{\psi_{\beta}\right\}\right\rangle$ and fiber $F^{\prime}$. An overlay transformation is a pair of maps $f: B \rightarrow B^{\prime}, \bar{f}: E \rightarrow \dot{E}^{\prime}$

(i) $p^{\prime} f=f p$.

(ii) There is a refinement $\left\langle\left\{W_{\gamma}\right\},\left\{Q_{\gamma}\right\}\right\rangle$ of $\left\langle\left\{U_{\alpha}\right\},\left\{\varphi_{\alpha}\right\}\right\rangle$ such that for each $\gamma$ there is a $\beta$ such that $f\left(W_{\gamma}\right) \subseteq V_{\beta}$ and a map $\sigma_{\gamma}: F \rightarrow F^{\prime}$ such that for each $x \in W_{\gamma}$ $\bar{f}\left(\varrho_{y}(x, t)\right)=\psi_{\beta}\left(f(x), \sigma_{y}(t)\right)$.

Two overlays with the notation above are said to be isomorphic if there are overlay transformations
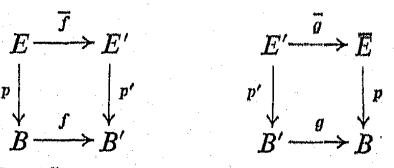

such that $\bar{g} \bar{f}=I_{E}$ and $\bar{f} \bar{g}=I_{E^{\prime}}$

III. 13. Proposition. Suppose $p: E \rightarrow B$ and $p^{\prime}: E^{\prime} \rightarrow B^{\prime}$ are overlays, and $\tilde{p}: \tilde{E} \rightarrow \mathscr{E}(B)$ and $\tilde{p}^{\prime}: \tilde{E}^{\prime} \rightarrow \mathscr{E}\left(b^{\prime}\right)$ are their respective associated $\mathscr{E}$-coverings. Suppose $(f, f)$ is a covering transformation from $p: E \rightarrow B$ to $p^{\prime}: E^{\prime}-B^{\prime}$. Then $\left(f, f^{\prime}\right)$ can be extended to an $\mathscr{E}$-covering space transformation $(\mathscr{E}(f), \tilde{f})$ from $\tilde{p}: \tilde{E} \rightarrow \mathscr{E}(B)$ to $\tilde{p}^{\prime}: \tilde{E}^{\prime} \rightarrow \mathscr{E}\left(B^{\prime}\right)$ if and only if $(f, \tilde{f})$ is an overlay transformation.

Proof. The proof is straightforward using the technique of Proposition III. 11.

Next, we wish to consider the converse of Corollary III. 10. Suppose $\tilde{p}: \tilde{E} \rightarrow \mathscr{E}(B)$ is an $\mathscr{E}$-covering space. Let $E=\tilde{p}^{-1}\left({ }^{*} B\right)$ and $p=\left.\tilde{p}\right|_{E}$. Notice $E$ and $p$ are internal and $p: E \rightarrow{ }^{*} B$ is a *overing space. We wish to known if there is a standard overlay $q: H \rightarrow B$ such that $p: E \rightarrow{ }^{*} B$ and ${ }^{*} q:{ }^{*} H \rightarrow{ }^{*} B$ are isomorphic. This is not always true. Consider the following examples.

IIr. 14. Examples. (i) Let $S$ denote the unit circle in the complex plane and consider the *covering space $p:{ }^{*} S \rightarrow * S$ given by $p(z)=z^{v}$ where $\nu$ is a fixed infinite positive integer. Thus, $p$ : ${ }^{*} S \rightarrow * S$ is a $y$-fold *covering of ${ }^{*} S$ which has an obvious extension to $\mathscr{E}(S)$ and which clearly has no standard counterpart. This example shows that we must at least require that the fiber $p^{-1}(b)$ be standard. However, the following example shows that this condition by itself is not sufficient.

(ii) Let $B$ be the figure eight and construct a *covering space $p: E \rightarrow^{*} B$ by covering the right hand loop with countably many disjoint $v$-fold *coverings and stringing them together with the universal *covering $* \boldsymbol{R} \rightarrow * S$ over the left hand loop as pictured below with $v=2$. Notice the fiber is $* Z$ but if $v$ is infinite $p: E \rightarrow \rightarrow^{*} B$ has no standard counterpart.

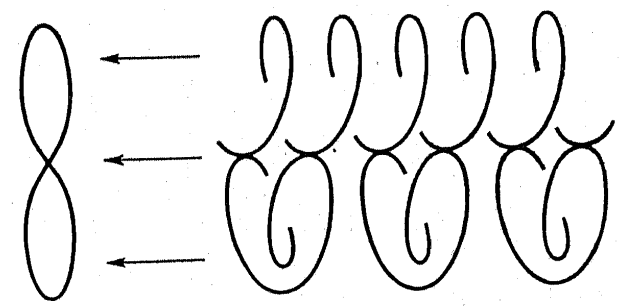

In order to formulate a sufficient condition for the converse of Corollary III. 10 we need some machinery.

III. 15. LemmA. Suppose $\tilde{p}: \tilde{E} \rightarrow \mathscr{E}(B)$ is an $\mathscr{E}$-covering space. Let $E=\tilde{p}^{-1}\left({ }^{*} B\right)$ and $p=\left.\tilde{p}\right|_{E}$. Then there is a *overlay structure $\left\langle^{*}\left\{U_{\alpha}\right\},\left\{\varphi_{\alpha}\right\}\right\rangle$ on $p: E \rightarrow B$ such that $*\left\{U_{\alpha}\right\}$ is a standard open cover of *B.

Proof. We will show there is a standard continuous function $f: B \rightarrow(0,1]$ such that the standard open covering $\left\{U_{x}\right\}_{x \in B}$ where $U_{x}=\{y \in B \mid d(x, y)<f(x)\}$ will do.

First let $T$ be the set of all internal *continuous functions $g: * B \rightarrow *(0,1]$ such that

(i) For each $x \in *^{*} B$, if $U_{x}=\left\{y \in *^{*} B \mid{ }^{*} D(x, y)<g(x)\right\}$ then $p^{-1}\left(U_{x}\right)$ can be written as a disjoint union of open sets $V_{x t}$ such that for each $x, t,\left.p\right|_{V x t}: V_{x t} \rightarrow U_{x}$ is

(ii) For each $x, y \in{ }^{*} B$ if $U_{x} \cap U_{y} \neq \emptyset$ then for each $t$ there is a unique $s$ such that $V_{x 1} \cap V_{y s} \neq \varnothing$. In other words $T$ is the set of all internal *continuous functions 
$g:{ }^{*} B \rightarrow *(0,1]$ such that there is a *overlay structure $\left\langle *\left\{U_{x}\right\},\left\{\varphi_{x}\right\}\right\rangle$ with $U_{x}=\left\{y \in *^{*} B \mid d(x, y)<g(x)\right\}$.

Now, consider the internal relation $R(f, g)$ defined by $g \notin T$ and $0<g \leqslant f$. If there is no standard $f \in T$ this relation is finitely satisfiable for standard continuous $f: B \rightarrow(0,1]$ and, hence, since $* \mathscr{M}$ is an enlargement there is an internal *continuous $\sigma:{ }^{*} B \rightarrow *(0,1]$ such that $\sigma \notin T$ and for all standard continuous $f: B \rightarrow(0,1] \sigma<*$. Let $V=\left\{x \in * X \mid \exists b \in *^{*} d(x, b)<\sigma(b)\right\}$. Let $E_{V}=\tilde{p}^{-1}(V)$ and $p^{\prime}=\left.\tilde{p}\right|_{E_{V}}$. Now the argument of Proposition III. 11 (i) shows $p^{\prime}: E_{V} \rightarrow V$ has a *overlay structure $\left\langle\left\{V_{b}\right\},\left\{\varphi_{b}\right\}\right\rangle$ with $V_{b}=\{x \in V \mid * d(x, b)<\sigma(b)\}$ which restricts to an overlay structure $\left\langle\left\{U_{b}\right\},\left\{\varphi_{b}\right\}\right\rangle$ with $U_{b}=\left\{x \in *^{*} B \mid * d(x, b)<\sigma(b)\right\}$. Hence $\sigma \in T$ and this contradiction completes the proof.

III. 16. Construction. Suppose $\tilde{p}: \tilde{E} \rightarrow \mathscr{E}(B)$ is an $\mathscr{E}$-covering space. By the preceding lemma and paracompactness we can find a *overlay structure $\left\langle *\left\{U_{\alpha}\right\},\left\{\varphi_{\alpha}\right\}\right\rangle$ such that $\left\{U_{\alpha}\right\}$ is a standard locally finite open cover of $B$. Given such a *overlay structure an admissible sequence is a sequence $\alpha_{1}, \alpha_{2}, \ldots, \alpha_{k}$ such that

(i) $b \in U_{\alpha_{1}} \cap U_{\alpha_{k}}$,

(ii) for $1 \leqslant i \leqslant k-1 \quad U_{\alpha_{i}} \cap U_{\alpha_{i+1}} \neq \varnothing$.

Let $\mathscr{A}$ be the set of all admissible sequences and define $\Phi: * \mathscr{A} \rightarrow H(F)$, where $H(F)$ is the set of homeomorphisms of $F$, by $\Phi\left(\alpha_{1}, \alpha_{2}, \ldots, \alpha_{v}\right)=g_{\alpha_{\nu}-1 \alpha_{\nu}} \ldots g_{\alpha_{2} \alpha_{3}} g_{\alpha_{1} \alpha_{2}}$ using the notation of Definition III. 1.

III. 17. Definition. An $\mathscr{E}$-covering space $\tilde{p}: \tilde{E} \rightarrow \mathscr{E}(B)$ is said to be costandard if there is a *overlay structure as above, a standard set $H$ and an internal bijection $\psi: F \rightarrow{ }^{*} H$ such that the mapping $\gamma:{ }^{*} \mathscr{A} \rightarrow{ }^{*} H(H)$ defined by $\gamma(s)=\psi \Phi(s) \psi^{-1}$ is standard.

A straightforward argument shows that an $\mathscr{E}$-covering space associated with a given standard overlay $p: E \rightarrow B$ is costandard. This condition is thus an appropriate one for the converse of Corollary III. 10 .

III. 18. THEOREM. If $\tilde{p}: \tilde{E} \rightarrow \mathscr{E}(B)$ is a costandard $\mathscr{E}$-covering space there is a standard overlay $q: K \rightarrow B$ such that ${ }^{*} q:{ }^{*} K \rightarrow{ }^{*} B$ is isomorphic to $p: E \rightarrow{ }^{*} B$ where $E=\tilde{p}^{-1}\left({ }^{*} B\right)$ and $p=\left.\tilde{p}\right|_{E}$.

Proof. We continue using the notation established above.

By a straightforward connectedness argument, for each standard $\alpha$ there is a standard sequence $\alpha_{1}, \alpha_{2}, \ldots, \alpha_{k}$ such that

(i) $b \in U_{\alpha_{1}}, \alpha_{k}=\alpha$,

(ii) for $1 \leqslant i \leqslant k-1 \quad U_{\alpha_{i}} \cap U_{\alpha_{i+1}} \neq \varnothing$.

Fix such a sequence for each $\alpha$. Given the sequence $\alpha_{1}, \alpha_{2}, \ldots, \alpha_{k}$ for $\alpha$ and the sequence $\beta_{1}, \beta_{2}, \ldots, \beta_{n}$ for $\beta$ if $U_{\alpha} \cap U_{\beta} \neq \varnothing$, notice the sequence

$$
s_{\alpha \beta}=\alpha_{1}, \alpha_{2}, \ldots, a_{k}, \beta_{n}, \ldots, \beta_{2}, \beta_{1}
$$

is in $\mathscr{A}$. Let $\varphi_{\alpha \beta}=\gamma\left(s_{\alpha \beta}\right)$.

Let $K^{\prime}$ be the disjoint union of the sets $U_{\alpha} \times H$. If $(x, t) \in U_{\alpha} \times H$ and $(x, s) \subseteq U_{\beta} \times H$ we say $(x, t) \sim(x, s)$ provided $s=\varphi_{\alpha \beta}(t)$. Define $\Gamma:{ }^{*} K^{\prime} \rightarrow E$ as follows. If $(x, t) \in U_{\alpha} \times * H$ and $\alpha_{1}, \alpha_{2}, \ldots, a_{v}$ is the sequence for $\alpha, \Gamma(x, t)$ $=\varphi_{\alpha_{\nu}}\left(x, \Phi\left(\alpha_{1}, \alpha_{2}, \ldots, a_{\nu}\right)(t)\right)$.

Notice if $(x, t) \in U_{\alpha} \times * H$ and $(x, s) \in U_{\beta} \times * H$ then $(x, t) \sim(x, s)$ if and only if $\Gamma(x, t)=\Gamma(x, s)$. Now $K=K^{\prime} / \sim$ and $\Gamma$ induces an isomorphism ${ }^{*} K \rightarrow E$.

III. 19. Remarks. The condition that $\tilde{p}: \tilde{E} \rightarrow \mathscr{E}(B)$ be costandard seems somewhat ad hoc at this point. If $B$ is compact (or, if the open covering $\left\{U_{\alpha}\right\}$ is finite) we can weaken this condition to - for each $s \in \mathscr{A}, \psi \Phi(s) \psi^{-1}$ is standard. However, it is easy to construct an $\mathscr{E}$-covering $\tilde{p}: \tilde{E} \rightarrow \mathscr{E}(B)$ where $B$ is not compact which satisfies this weaker condition but does not extend any standard overlay. In particular cases the costandardness condition may be very easy to check.

In view of our work thus far, standard overlays, $p: E \rightarrow B$, are in bijective correspondence with costandard $\mathscr{E}$ mcovering spaces, $\tilde{p}: \tilde{E} \rightarrow \mathscr{E}(B)$. Thus, the next step is the classification of $\mathscr{E}$-covering spaces. From now on we work in the category $\mathscr{E}$. The basic facts of covering space theory carry over unchanged to this category as follows.

III. 20. Definition. Suppose $p: E \rightarrow B$ is an $\mathscr{E}$-covering space and $f: X \rightarrow B$. A lifting for $f$ is a map $f: X \rightarrow E$ such that $p \bar{f}=f$.

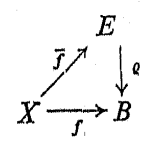

III. 21. BASIC LEMMA. Suppose $p: E \rightarrow B$ is an $\mathscr{E}$-covering, $X$ is path-connected and $f: X \rightarrow B$. If $f_{1}$ and $f_{2}$ are two liftings of $f$ and for some $x \in X, f_{1}(x)=f_{2}(x)$ then $f_{1}=f_{2}$.

Proof. Completely analogous to the usual proof [14].

III. 22. Proposition. Suppose $p: E \rightarrow B$ is an $\mathscr{E}$-covering space, $\omega:{ }^{*} I \rightarrow B$ and $e \in p^{-1}(\omega(0))$. Then there is a unique lifting $\bar{\omega}$ of $\omega$ such that $\bar{\omega}(0)=e$.

Proof. Completely analogous to the usual proof [14].

III. 23. Propostrion. Suppose $p: E \rightarrow B$ is an $\mathscr{E}$-covering space, $f:{ }^{*} I^{2} \rightarrow B$ and $e \in p^{-1}(f(0,0))$. Then there is a unique lifting $f$ of $f$ such that $f(0,0)=e$. Proof. Completely analogous to the usual proof [14].

III. 24. Coroltary. Suppose $p: E \rightarrow B$ is an $\mathscr{E}$-covering space and $p(e)=b$. Then $p_{*}: \pi_{1}(E, e) \rightarrow \pi_{1}(B, b)$ is injective.

Proof. Completely analogous to the usual proof [14].

III. 25. Corollary. Suppose $p: E \rightarrow B$ is an 8 -covering space, $b \in B$ and $\in \in F=p^{-1}(b)$. Suppose $\omega \in \Omega(B, b)$ and let $\bar{\omega}$ be the unique lifting of $\omega$ such that $\bar{\omega}(0)=e$. Then $\bar{\omega}$ is a loop $(i . e . \bar{\omega}(1)=e)$ if and only if $[\omega] \in p_{*}\left(\pi_{1}(E, e)\right)$. Thus, there is a bijection $\tau: \pi_{1}(B, b) / p_{*}\left(\pi_{1}(E, e)\right) \rightarrow F$ defined by $\tau([\omega])=\bar{\omega}(1)$, where $\pi_{1}(B, b) / p_{*}\left(\pi_{1}(E, e)\right)$ is the set of all cosets of the form $\left[p_{*}\left(\pi_{1}(E, e)\right)\right] x$ for some $\pi_{1}(B, b) / p_{*}\left(\pi_{1}(B, b)\right.$.

Proof. Completely analogous to the usual proof [14]. 
The following technical property is needed to establish the usual theorem about liftings of maps $f: X \rightarrow B$.

III. 26. Definition. An $\mathscr{E}$-space $X$ is said to satisfy property (P) if for every internal set $A \subseteq X$ there is an internal set $A^{\prime}, A \subseteq A^{\prime} \subseteq X$ which is *path-connected and *locally-path-connected.

III. 27. LEMMA. (i) If $X$ is connected, $\mathscr{E}(X)$ satisfies property (P).

(ii) If $p: E \rightarrow B$ is a (standard) overlay and $\tilde{p}: \tilde{E} \rightarrow \mathscr{E}(B)$ is its associated $\&$-covering space then $\tilde{E}$ satisfies property $(\mathrm{P})$.

Proof. (i) is an immediate consequence of Theorem II. 12.

(ii) If $A \subseteq \tilde{E}$ is internal, we may assume ${ }^{*} E \subseteq A$. Since $\tilde{p}$ is an $\mathscr{E}$-map, $\tilde{p}(A)$ is internal and by (i) there is an internal, *path-connected, *locally-path-connected set $C, \tilde{p}(A) \subseteq C \subseteq \mathscr{E}(B) . A^{\prime}=\tilde{p}^{-1}(A)$ is easily seen to be the desired set.

III. 28. Theorem. Suppose $p: E \rightarrow B$ is an $\mathscr{E}$-covering space and $X$ is a space which tatisfies property (P). Suppose $x \in X, b \in B, e \in p^{-1}(b)$ and $f:(X, x) \rightarrow(B, b)$. Then shere is a lifting $\vec{f}$ of $f$ such that $\vec{f}(x)=e$ if and only if $f_{*}\left(\pi_{1}(X, x)\right) \subseteq p_{*}\left(\pi_{1}(E, e)\right)$.

$$
(X, x) \stackrel{(E, e)}{\longrightarrow} \underset{s}{\longrightarrow}(B, b)
$$

Proof. The proof is entirely analogous to the usual proof [14, Theorem 2.4.5] with the observation that the construction of $f$ is internal on internal subsets of $X$ in view of property (P).

III. 29. Corollary. Suppose $p: E \rightarrow B$ and $p^{\prime}: E^{\prime} \rightarrow B$ are two 8 -coverings, and $E$ and $E^{\prime}$ satisfy property $(\mathrm{P})$. Suppose $b \in B, e \in p^{-1}(b)$ and $e^{\prime} \in\left(p^{\prime}\right)^{-1}(b)$. Then these two $\mathscr{E}$-covering spaces are equivalent in the sense that there is a homeomorphism $\varphi:(E, e) \rightarrow\left(E^{\prime}, e^{\prime}\right)$ such that $p^{\prime} \varphi=p$ if and only if $p_{*}\left(\pi_{1}(E, e)\right)=p_{*}^{\prime}\left(\pi_{1}\left(E^{\prime}, e^{\prime}\right)\right)$.

Proof. Immediate.

III. 30. Corollary. Suppose $p: E \rightarrow B$ and $p^{\prime}: E^{\prime} \rightarrow B$ are two (standard) overlays. Suppose $b \in B, e \in p^{-1}(b)$, and $e^{\prime} \in\left(p^{\prime}\right)^{-1}(b)$. Then $\Gamma: E \rightarrow B$ and $p^{\prime}: E^{\prime} \rightarrow B$ are isomorphic overlays via maps $f:(E, e) \rightarrow\left(E^{\prime}, e^{\prime}\right)$ and $\bar{g}:\left(E^{\prime}, e^{\prime}\right) \rightarrow(E, e)$ if and only if $p_{*}\left(\pi_{1}(\tilde{E}, e)\right)=p_{*}\left(\pi_{1}\left(\tilde{E}, e^{\prime}\right)\right)$ in $\pi_{1}(\mathscr{E}(B), b)$ (using the notation of III. 12 and III. 13).

Proof. This would be immediate from Corollary III. 29 and Proposition III. 13 except that Proposition III. 13 is not in terms of the points $e, e^{\prime}$. However, since the maps $\tilde{f}, \tilde{g}$ extend $\bar{f}, \bar{g}$ this implies $\tilde{f}(e)=\tilde{f}(e)=e^{\prime}$ and $\tilde{g}\left(e^{\prime}\right)=\bar{g}\left(e^{\prime}\right)=e$. Conversely, given internal $\mathscr{E}$-covering space isomorphisms $f:\left(\tilde{E}^{\prime}, e^{\prime}\right) \rightarrow(\tilde{E}, e)$ from Corollary III. 29 there are standard covering space isomorphisms $h:(E, e) \rightarrow\left(E^{\prime}, e^{\prime}\right)$ and $k:\left(E^{\prime}, e^{\prime}\right) \rightarrow(E, e)$. Then the Basic Lemma III. 21 implies that $\tilde{f}$ extends $* h$
and $\tilde{g}$ extends $* k$.

Corollary III. 30 solves half of the classification problem. Next, we turn to the problem of constructing an $\mathscr{E}$-covering space $p: E \rightarrow B$ such that $p_{*}\left(\pi_{1}(E, e)\right)$ is
a given subgroup of $\pi_{1}(B, b)$. It is clear from Example III. 5 that not every such subgroup gives rise to an $\mathscr{E}$-covering space. In particular, the trivial subgroup of $\pi_{1}\left(\mathscr{E}\left(S^{\omega}\right), b\right)$ does not. The following condition seems appropriate.

III. 31. Definitron. A subgroup $G$ of $\pi_{1}(B, b)$ is said to be $Q$-subgroup provided

(Q1) For every internal subset $A$, such that $b \in A \subseteq B, i_{*}^{-1}(G)$ is an internal subgroup of $\pi_{1}(A, b)$, where $i$ denotes the inclusion $A \subseteq B$.

(Q2) There is an internal set $A, b \in A \subseteq B$ such that

$$
\pi_{1}(A, b) \stackrel{i_{m}}{\rightarrow} \pi_{1}(B, b) \stackrel{Q}{\rightarrow} \pi_{1}(B, b) / G
$$

$\varrho i_{*}$ is onto where $\pi_{1}(B, b) / G$ is the set of cosets of the form $G x$ and $\varrho$ is the obvious surjection.

III. 32. Proposition. Suppose $p: E \rightarrow B$ is an $\mathscr{E}$-covering, $e \in E, b \in B$ and $p(e)=b$. Then $p_{*}\left(\pi_{1}(E, e)\right)$ satisfies condition $(\mathrm{Q} 1)$ of Definition III. 31. If $B=\mathscr{E}(X)$ for some standard space $X$ and $p: E \rightarrow B$ is the $\mathscr{E}$-covering space associated to a standard overlay then $p_{*}\left(\pi_{1}(E, e)\right)$ is' a $Q$-subgroup of $\pi_{1}(B, b)$.

Proof. (i) Suppose $b \in A \subseteq B$ and $A$ is internal. Let $E_{A}=p^{-1}(A)$ and $p^{\prime}=\left.p\right|_{E_{A}}$. Then $p^{\prime}: E_{\Lambda} \rightarrow A$ is an internal *covering space, so $p_{*}^{\prime}\left(\pi_{1}\left(E_{A}, e\right)\right)$ is an internal subgroup of $\pi_{1}(A, b)$. Let $i$ denote the inclusion map $A \rightarrow B$. By Corollary III. 25 if $[\omega] \in \pi_{1}(A, b)$ then, $[\omega] \in p_{*}^{\prime}\left(\pi_{1}\left(E_{A}, e\right)\right) \leftrightarrow \omega$ lifts to a loop $\bar{\omega}$ such that $\bar{\omega}(0)=e$ in $E_{A} \leftrightarrow[i \omega] \in p_{*}\left(\pi_{1}(E, e)\right)$. Thus, $p_{*}^{\prime}\left(E_{A}, e\right)=i_{*}^{-1}\left(p_{*}\left(\pi_{1}(E, e)\right)\right)$ and $i_{*}^{-1}\left(p_{*}\left(\pi_{1}(E, e)\right)\right)$ is internal.

(ii) Now suppose $B=\mathscr{E}(X)$ and $p: E \rightarrow B$ is the $\mathscr{E}$-covering associated to some standard overlay. By the proof of Lemma III. 27 there is an internal set $A$ such that $* X \subseteq A \subseteq B$ and both $A$ and $E_{A}=p^{-1}(A)$ are path connected. Let $F=p^{-1}(b)$. For each $e^{\prime} \in F$ there is a path $\omega_{e^{\prime}}$ in $E_{A}$ such that $\omega_{e^{\prime}}(0)=e$ and $\omega_{e^{\prime}}(1)=e^{\prime}$. By Corollary III. 25 the map $\tau: \pi_{1}(B, b) \rightarrow F$ defined by

$$
\tau([v])=\bar{v}(1)
$$

where $\bar{v}$ is the unique lifting of $v$ such that $v(0)=e$ establishes a bijection between $\pi_{1}(B, b) / p_{*}\left(\pi_{1}(E, e)\right)$ and $F$. Hence, since $\tau\left(p \omega_{e^{\prime}}\right)=e^{\prime}$ the map $f$ tefined by

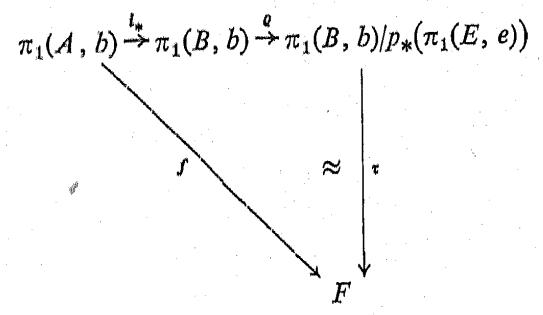

is surjective so $Q i_{*}$ is also surjective completing the proof.

The construction of an 8 -covering space corresponding to a given subgroup 
is essentially the same as in the standard case. However, as in the case of Theorem III. 28 a technical property is necessary to stay in the category $\mathscr{E}$.

III. 33. Definttion. An $\mathscr{E}$-set $B$ is said to satisfy property (S) provided for every internal set $A \subseteq B$ there is an internal set $A^{\prime}, A \subseteq A^{\prime} \subseteq B$ such that $A^{\prime}$ is pathwise connected, locally pathwise connected, and locally simply-connected. Notice, by Lemma II. 12 if $X$ is connected, $\mathscr{E}(X)$ satisfies property (S).

III. 34. Construction. Suppose $B$ is an internal, connected, locally path connected and locally simply connected space, $b \in B$, and $G$ is an internal subgroup of $\pi_{1}(B, b)$.

Let $\Omega=\{\omega: * I \rightarrow B \mid \omega(0)=b\}$.

If $u, v \in \Omega$ we write $u \simeq v$ provided

(i) $u(1)=v(1)$ and

(ii) $[q] \in G$ where $q$ is the loop defined by

$$
q(t)=\left\{\begin{array}{lll}
u(2 t) & \text { if } & 0 \leqslant t \leqslant \frac{1}{2}, \\
v(2-2 t) & \text { if } & \frac{1}{2} \leqslant t \leqslant 1 .
\end{array}\right.
$$

Define $p^{\prime}: \Omega \rightarrow B$ by $p^{\prime}(\omega)=\omega(1)$. Let $E=\Omega / \simeq$ and let $p: E \rightarrow B$ be the map induced by $p^{\prime}$. Let $e \in E$ be the equivalence class containing the constant path $\omega(t)=b$.

Then $p: E \rightarrow B$ is a covering space and $p_{*}\left(\pi_{1}(E, e)\right)=G$.

Proof. This is just the nonstandard form of the usual existence theorem for covering spaces [14].

This theorem then allows us to prove the following theorem.

III. 35. Theorem. Suppose $B$ is $a \mathscr{E}$-set with property (S), $b \in B$ and $G \subseteq \pi_{1}(B, b)$ is a $Q$-subgroup. Then there is an $\mathscr{E}$-covering space $p: E \rightarrow B$ and a point $e \in p^{-1}(b)$ such that $p_{*}\left(\pi_{1}(E, e)\right)=G$.

Proof. Essentially the construction is the same as III. 34 except that $B$ is not internal and we wish to stay within the category $\mathscr{E}$. This forces our construction to be more circuitous.

Let $X$ be a standard topological space such that $B \subseteq * X$. Let $\Omega=\{\langle A, H\rangle \mid A$ is internal, path connected, locally path connected and locally simply connected; $b \in A \subseteq \subseteq^{*} X$ and $H$ is an internal subgroup of $\left.\pi_{1}(A, b)\right\}$. Notice $\Omega$ is internal. In fact, $\Omega$ is the nonstandard extension of a standard set.

For each $\langle A, H\rangle \in \Omega$ let

$$
E_{\langle A, H\rangle}^{\prime}=\{\omega: I \rightarrow A \mid \omega(0)=b\}
$$

If $u, v \in E_{\langle A, H\rangle}^{\prime}$ we write $u \simeq v$ provided

(i) $u(1)=v(1)$ and

(ii) $[q] \in H$ where $q$ is the loop

$$
q(t)=\left\{\begin{array}{lll}
u(2 t) & \text { if } & 0 \leqslant t \leqslant \frac{1}{2} \\
v(2-2 t) & \text { if } & \frac{1}{2} \leqslant t \leqslant 1
\end{array}\right.
$$

Now let $E_{\langle A, I I\rangle}=E_{\langle A, I I\rangle}^{\prime} / \simeq$, and give $E_{\langle A, H\rangle}$ the quotient topology. Let $p_{\langle A, I I\rangle}: E_{\langle A, I I\rangle} \rightarrow A$ be the obvious map.

Now if $\langle A, H\rangle,\left\langle A^{\prime}, H H^{\prime}\right\rangle \in \Omega$ we write $\langle A, H\rangle \leqslant\left\langle A^{\prime}, H^{\prime}\right\rangle$ provided

(i) $A \subseteq A^{\prime}$,

(ii) $i_{*}^{-1}\left(H^{\prime}\right)=H$ where $i$ is the inclusion $A C \rightarrow A^{\prime}$.

Notice, if $\langle A, H\rangle \leqslant\left\langle A^{\prime}, H^{\prime}\right\rangle$ then $E_{\langle A, H\rangle}^{\prime} \subseteq E_{\left\langle A^{\prime}, H^{\prime}\right\rangle}^{\prime}$ and if $u, v \in E_{\langle A, H\rangle}^{\prime}$ then $u \simeq v$ in $E_{\langle A, I I\rangle}^{\prime} \leftrightarrow u \simeq v$ in $E_{\left\langle A^{\prime}, I^{\prime}\right\rangle}^{\prime}$.

Now, let $E^{\prime \prime}$ be the disjoint union.

$$
E^{\prime \prime}=\bigcup_{\langle A, I Y\rangle \in \Omega} E_{\langle A, I I\rangle}
$$

Now suppose $u, v \in E^{\prime \prime}$ where $u \in E_{\langle A, H\rangle}, v \in E_{\left\langle A^{\prime}, H^{\prime}\right\rangle}$. We say $u \simeq v$ provided there is a $\left\langle A^{\prime \prime}, H^{\prime \prime}\right\rangle \in \Omega$ such that

(i) $\langle A, H\rangle \leqslant\left\langle A^{\prime \prime}, H^{\prime \prime}\right\rangle$ and $\left\langle A^{\prime}, H^{\prime}\right\rangle \leqslant\left\langle A^{\prime \prime}, H^{\prime \prime}\right\rangle$ and

(ii) considered as elements of $E_{\left\langle A^{\prime \prime}, H^{\prime \prime}\right\rangle} u \simeq v$.

Next, let $E^{\prime}=E^{\prime \prime} \mid \simeq$ with the quotient topology and $p^{\prime}: E^{\prime} \rightarrow * X$ be the obvious map. Notice for each $\langle A, H\rangle \in \Omega E_{\langle A, H\rangle}$ may be regarded as a subspace of $E^{\prime}$. Let $i_{\langle A, H\rangle}$ denote the inclusion $E_{\langle A, H\rangle} C_{\rightarrow} \rightarrow E^{\prime}$. Notice $E^{\prime}$ is internal. In fact $E^{\prime}$ is the nonstandard extension of $A$ standard set.

Recall that our original subgroup $G \subseteq \pi_{1}\left(B, b_{0}\right)$ was a $Q$-subgroup; so that for each internal $A$ such that $b \in A \subseteq B, H_{A}=i_{*}^{-1}(G) \subseteq \pi_{1}(A, b)$ is also internal where $i$ is the inclusion map $A C \rightarrow B$.

Finally, let

$E=\left\{x \in E^{\prime} \mid\right.$ there is an internal set $A$ s.t. $b_{0} \in A \subseteq B$ and $\left.x \in \operatorname{Im} i_{\left\langle A, H_{A}\right\rangle}\right\}$.

Notice, $E$ is our first external set and $E \subseteq E^{\prime}$ so $E$ is an $\mathscr{E}$-set. Let $p: E \rightarrow B$ be $\left.p^{\prime}\right|_{E}$. Let $e \in E$ be the equivalence class containing the constant path $\omega(t)=b$. We claim $p: E \rightarrow B$ is the soughtfor $\mathscr{B}$-covering.

Let $A$ be any internal subset of $B$. Since $B$ satisfies property (S) and $G$ is a $Q$-subgroup we may assume $A$ is pathwise connected, locally pathwise connected and locally simply connected and

$$
\pi_{1}(A, b) \stackrel{l_{4}}{\rightarrow} \pi_{1}(B, b) \stackrel{e}{\rightarrow} \pi_{1}(B, b) / G
$$

is onto. Since $G$ is a $Q$-subgroup $H=i_{*}^{-1}(G) \subseteq \pi_{1}(A, b)$ is internal. So $\langle A, H\rangle \in \Omega$. By construction III. $34 p_{\langle A, I\rangle}: E_{\langle A, H\rangle} \rightarrow A$ is a ${ }^{*}$ covering space, so all we need show is that $p^{-1}(A)=E_{\langle A, I T\rangle}$. Clearly $E_{\langle A, H\rangle} \subseteq p^{-1}(A)$. Now suppose $x \in p^{-1}(A)$. Let $u$ be a path in $A$ from $b$ to $p(x)$ and let $\tilde{u}$ be the unique lifting of $u$ to $E_{A}=p^{-1}(A)$ such that $\bar{u}(1)=x$. Let $e^{\prime}=\bar{u}(0)$. Since $Q l_{*}$ is onto there is a loop $v$ in $\Omega(A, b)$ such that if $\bar{v}$ is the unique lifting of $v$ to $E_{A}$ such that $\bar{v}(0)=e, \bar{v}(1)=e^{\prime}$. Let $q$ be the path in $A$ given by

$$
q(t)=\left\{\begin{array}{lll}
v(2 t) & \text { if } & 0 \leqslant t \leqslant \frac{1}{2} \\
u(2 t-1) & \text { if } & \frac{1}{2} \leqslant t \leqslant 1 .
\end{array}\right.
$$


Let $\bar{q}$ be the unique lifting of $q$ to $E_{A}$ such that $\bar{q}(0)=e$. In fact $\bar{q}$ can be represented by the path

$$
Q(t)(s)=q(s t) .
$$

Clearly $Q$ is a path in $E_{\langle A, H\rangle}$ and $x=\bar{q}(1)=Q(1) \in E_{\langle A, H\rangle}$ completing the proof

Finally, we define a $Q$-subgroup $G \subseteq \pi_{1}(\mathscr{E}(B), b)$ to be costandard if the corresponding $\mathscr{E}$-covering space is costandard as follows.

III. 36. Definition. Suppose $G \subseteq \pi_{1}(\mathscr{E}(B), b)$ is a $Q$-subgroup and $p: E \rightarrow \mathscr{E}(B)$ is the corresponding $\mathscr{E}$-covering space. Let $e \in E$ be as above. $G$ is said to be costandard if

(i) $p: E \rightarrow \mathscr{E}(B)$ is costandard and

(ii) using the notation of Definition III. $17, \psi$ may be chosen so that $\psi(e)$ is standard.

The results of this section can then be summarized by

III. 37. THEOREM. Equivalence classes of overlay structures $p: E \rightarrow B$ with disw tinguished points $e$ are in bijective correspondence with costandard, $Q$-subgroups of $\pi_{1}(\mathscr{E}(B), b)$.

\section{References}

[1] K. Borsuk, Concerning homotopy properties of compacta, Fund. Math. 62 (1968), pp. 223-254.

[2] - Theory of Shape, Aarhus Lecture Note Series No. 28, 1970.

[3] D. E. Chris tie, Net homotopy for compacta, Trans. Amer. Math. Soc. 56 (1944), pp. 275-308.

[4] J. Dugundji, ANR and local connectivity in arbitrary metric spaces, Comp. Math. 13 (1958), pp. 229-246.

[5] - Topology, Boston 1968.

[6] R. H. Fox, On shape, Fund. Math. 74 (1972), pp. 47-71.

[7] W. Henson, The isomorphism property in nonstandard analysis and its use in the theory of Banach spaces, J. Symb. Logic 39 (1974), pp. 717-773. "

[8] S. T. Hu, Homotopy Theory, New York 1959.

[9] K. Kuratowski, Topology, Vol. I, New York-London-Warszawa 1966.

10] M. Machover and J. Hirschfeld, Lectures on Non-standard Analysis, Lecture Notes in Mathematics 94, Berlin 1969.

[11] M. C. Mc Cord, Non-standard analysis and homology, Fund. Math. 74 (1972), pp. 21-28

[12] T. Moore, On Fox's Theory of Overlays.

[13] A. Robinson, Non-standard Analysis, Amsterdam 1966

[14] E. Spanier, Algebraic Topology, New York 1966.

15] F. Watten berg, Nonstandard topology and extensions of monad systems to infinite point J. Symb. Logic 36 (1971), pp. 463-476.

[16] - Monads of infinite points and finite product spaces, Trans. Amer. Math. Soc. 176 (1973) pp. $351-368$.

\section{Collectionwise normality and absolute retracts}

\section{T. Przymusiński (Warszawa)}

- Abstract. In this paper we present a generalization and a unification of the classical theorems concerning absolute retracts for metrizable spaces, which were proved in the early fifties by Arens, Dowker, Hanner and Michael.

To illustrate possible applications of the obtained results we derive from them a generalization of Borsuk's homotopy extension theorem, which is a slight strengthening and reformulation of the recent result of Morita and Starbird.

$\S 1$. Introduction. Let $Q$ be an arbitrary class of topological spaces. A space $X \in Q$ is an absolute retract for the class $Q$ (briefly: an $\operatorname{AR}(Q)$-space) if for every space $Z \in Q$ containing $X$ as a closed subspace there exists a continuous retraction of $Z$ onto $X$. A topological space $X$ is an absolute extensor for the class $Q$ (an $\operatorname{AE}(Q)$-space) if for every space $Z \in Q$, its closed subspace $F$ and continuous mapping $f: F \rightarrow X$ there exists a continuous extension $f: Z \rightarrow X$ of $f$ onto $Z$.

Note, that in the definition of an absolute extensor we do not require $X$ to be a member of $Q$. A space $X \in Q$ is an absolute neighbourhood retract for the class $Q$ (an $\operatorname{ANR}(Q)$-space) if for every space $Z \in Q$ containing $X$ as a closed subspace there exists a neighbourhood $U$ of $X$ in $Z$ and continuous retraction of $U$ onto $X$. A topological space $X$ is an absolute neighbourhood extensor for the class $Q$ (an $\operatorname{ANE}(Q)$ space) if for every space $Z \in Q$, its closed subspace $F$ and a continuous mapping $f: F \rightarrow X$ there exists a neighbourhood $U$ of $F$ in $Z$ and a continuous extension $f: U \rightarrow X$ of $f$ onto $U$.

Absolute retracts for normal spaces are called briefly absolute retracts (AR-spaces). Similarly absolute extensors (AE-spaces), absolute neighbourhood retracs (ANR-spaces) and absolute neighbourhood extensors (ANE-spaces) are defined.

FACT 1 (The Tietze-Urysohn theorem). The real line $E$ and the unit interval $I=[0,1]$ are absolute extensors.

One can easily check that if a space $X$ belongs to $Q$ and is an $\operatorname{AE}(Q)$-space (resp. an $\operatorname{ANE}(Q)$-space), then $X$ is an $\operatorname{AR}(Q)$-space (resp. an $\operatorname{ANR}(Q)$-space). It turns out that in "good" classes of spaces the inverse implication holds.

FACr 2 (Hanner [12], Michael [15]). If $Q$ denotes the class of normal (resp. metrizable; resp. compact) spaces and if $X$ belongs to $Q$, then: 